\title{
Creation of an atrial septal defect in transposition of the great vessels
}

\author{
MER VYN S. GOTS MAN ${ }^{1}$ \\ From the Heart Unit, The Children's Hospital, Ladywood Road, Birmingham
}

Four-fifths of the infants with transposition of the great vessels die within the first year of life (Abbott, 1936). Fifty-two per cent. die within the first month and $86 \%$ are dead within six months (Keith, Neill, Vlad, Rowe, and Chute, 1953). This lesion in some form occurs in $20 \%$ of necropsies on patients with congenital heart disease (Keith, Rowe, and Vlad, 1958). In Birmingham, transposition of the great vessels is the commonest form of cyanotic congenital heart disease in patients admitted to the neonatal cardiac unit. The outcome is frequently fatal. The prognosis is worse if a ventricular septal defect is absent (Noonan, Nadas, Rudolph, and Harris, 1960) and the atrial septal defect is small (Mustard, Keith, Trusler, Fowler, and Kidd, 1964). Any form of therapy is therefore useful if it can prolong life until the patient has grown sufficiently to benefit from corrective surgery. Hanlon and Blalock (1948) and Fontana and Edwards (1962) found that the duration of survival is longest when both atrial and ventricular septal defects are present. The creation of an atrial septal defect therefore appears to be a rational form of palliative therapy (Ochsner, Cooley, Harris, and McNamara, 1961; Mustard, 1964; Mustard et al., 1964; Trusler, Mustard, and Fowler, 1964). Unfortunately, no detailed haemodynamic reports are available to indicate the effectiveness of creating an atrial septal defect, since cardiac catheterization in this group of patients is difficult and dangerous.

We shall describe the findings at cinéangiocardiography before and after the creation of an atrial septal defect in a seriously ill neonate who improved after operation.

\section{CASE REPORT}

A.C., a second child. was born at home at full term. Cyanosis was noted at birth. This deepened on the

1Present address: The Cardiac Clinic, Groote Schuur Hospital, Observatory, Cape Town, South Africa sixth day with a rapid, panting pattern of breathing, and the infant refused to feed.

The dyspnoeic, deeply cyanosed infant was ad- 을 mitted to hospital in gross heart failure on the eighth day of life. The eyelids and feet were oedematous $z$ and the liver enlarged $3 \mathrm{~cm}$. below the right costal margin. The child was hypothermic $\left(95^{\circ}\right.$ F.). The $\frac{\Phi}{3}$ heart was enlarged with a split second sound and a $\underset{\mathbb{D}}{\overrightarrow{0}}$ loud third heart sound. No murmurs were heard. The chest radiograph suggested transposition of the $\overrightarrow{\mathscr{Q}}$ great vessels (Fig. 1), a large heart with pulmonary of plethora, and a full left middle segment. An electrocardiogram showed right ventricular hypertrophy. The child had severe metabolic acidosis with alveolar hyperventilation $\left(p \mathrm{H} 7.25, \mathrm{PCO}_{2} 17 \mathrm{~mm}\right.$. $\mathrm{Hg}$, and standard bicarbonate $15.5 \mathrm{mEq} / \mathrm{l}$.).

The clinical picture of transposition of the great $\stackrel{\mathbb{Q}}{\Omega}$ vessels with an intact ventricular septum, hypoxia, $\underset{\vec{P}}{\overrightarrow{2}}$ hypothermia, and metabolic acidosis required urgent management. The cardiac failure was treated with digitalis and chlorothiazide, and the metabolic acidosis was corrected with Tris-buffer. The child's condition deteriorated.

A venous cinéangiocardiogram was performed. Tracings from the film sequence are shown in Figure $\stackrel{x}{x}$ 2. Figure $2 \mathrm{~A}$ shows the patient in the left anterior $\frac{0}{7}$ oblique position. A small quantity of contrast medium passed from the right to the left atrium $\delta$ across a small patent foramen ovale ('pony tail $३$ appearance'). Six frames later the foramen ovale was 0 seen again (Fig. 2B). Contrast medium then passed into the right ventricle and an aorta situated $\frac{D}{0}$ anteriorly with the aortic valve at a higher level than normal. Insufficient contrast passed to the left atrium $N$ and ventricle to fill these chambers or the pulmonary artery. The ductus arteriosus also filled but formed $\mathcal{O}$ a stagnant recess without the pulsatile appearance $\underset{\omega}{N}$ which is seen when the lumen is patent throughout $\sigma$ its length. This indicated that the pulmonary end of the ductus had closed. Figure $2 \mathrm{C}$ shows the corresponding appearance in the right anterior $\Phi^{\circ}$ oblique position. These angiocardiographic findings suggested that recent closure of the ductus arteriosus was responsible for the deterioration in clinical $\frac{\vec{D}}{\mathrm{D}}$ status. Urgent surgery was performed on the same $\stackrel{\odot}{\mathcal{P}}$ day and a Blalock-Hanlon procedure was carried out $\stackrel{\Phi}{\varrho}$ creating an atrial septal defect $4 \mathrm{~mm}$. by $1 \mathrm{~cm}$. in 


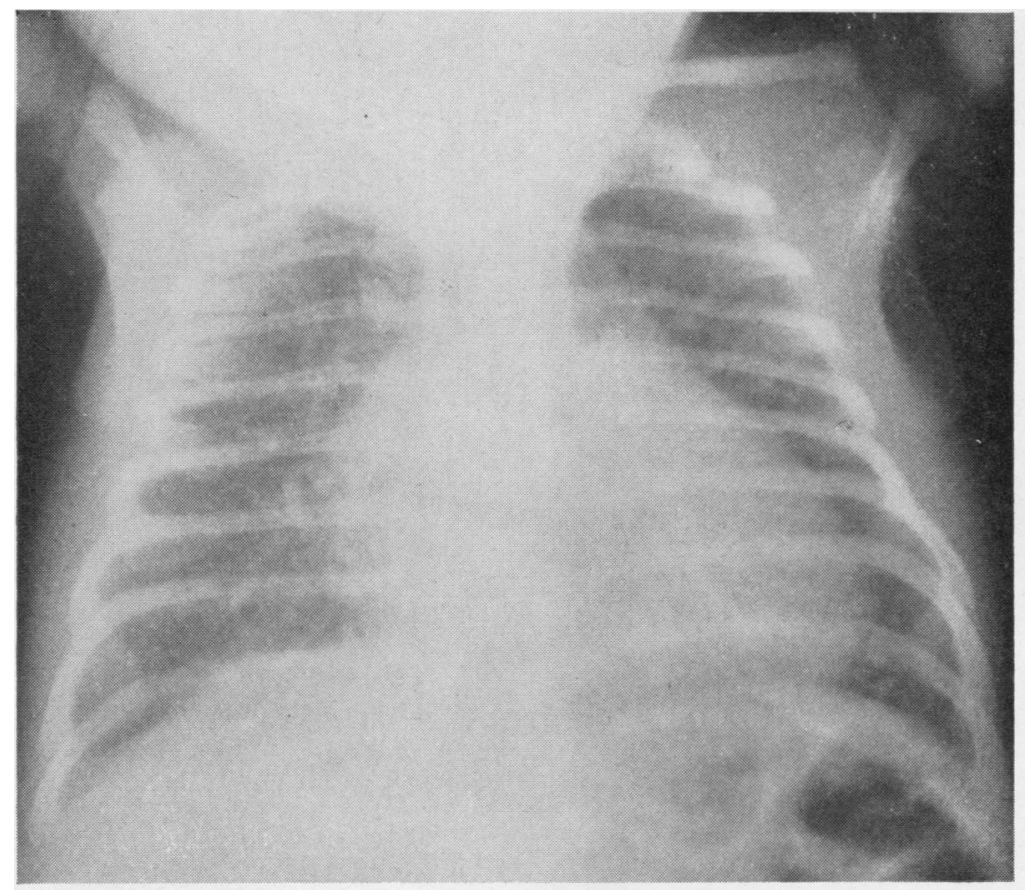

(a)

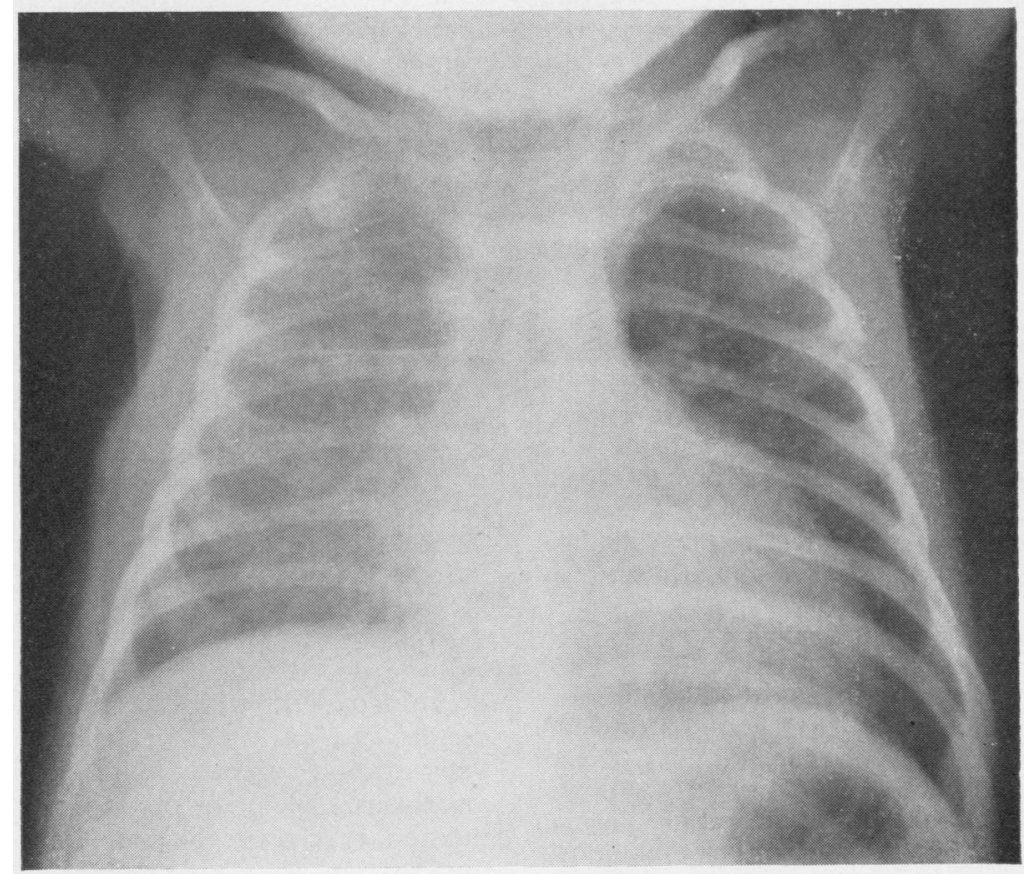

(b)

FIG. 1. Chest radiographs (a) before creation of the atrial septal defect. The heart was enlarged with gross pulmonary plethora; (b) two weeks after operation. The heart had become slightly smaller. 

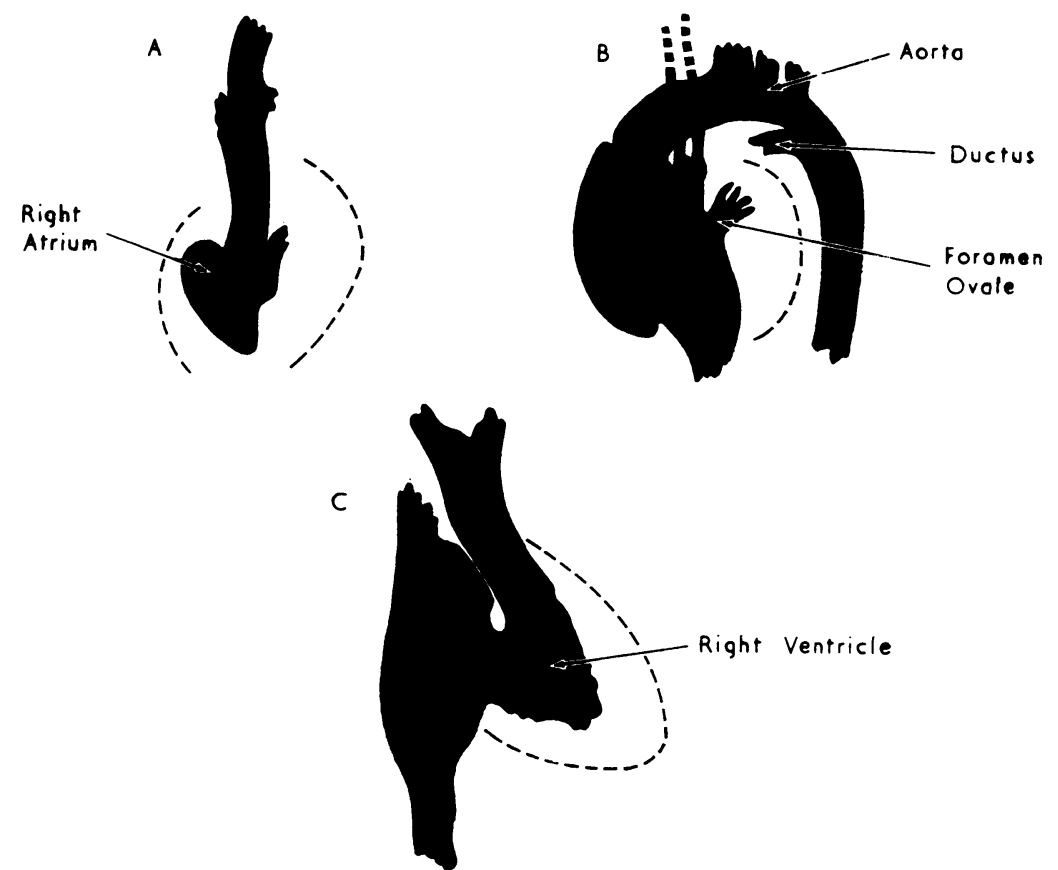

FIG. 2. Venous angiocardiography before operation (age 8 days). (A) Left anterior oblique position. Contras medium has entered a large right atrium and has passed into the left atrium through a small patent foramen ovale ('pon y® tail' appearance). The transseptal flow occurred only during atrial systole. (B) Left anterior oblique position-six $F$ frames later. Contrast passed into the right ventricle and then into an anteriorly situated aorta. The aortic valve lays in an abnormally high position. The foramen ovale was seen again. A stagnant column of contrast medium without 'to and fro' movement filled the aortic end of the ductus arteriosus. (C) Right anterior oblique position. This frame corresponds to Figure $2 B$. Transposition of the aorta was confirmed. Very little contrast medium entered the leff atrium. The left ventricle and pulmonary artery were not seen. There was inadequate mixing of the pulmonary an\$ systemic circuits with a small right to left shunt through the foramen ovale and no indication of the left to right shunt (if any).

size (Blalock and Hanlon, 1950) : Ochsner et al., 1961).

The post-operative period was complicated by retention of sputum and severe obstructive airways disease. The $\mathrm{PCO}_{2}$ increased to $56 \mathrm{~mm}$. $\mathrm{Hg}$. With prolonged intensive care the infant improved and after a fortnight the chest was normal. The cyanosis had also improved, and a week later the child was weaned from the incubator. No murmurs had appeared.

A second venous cinéangiocardiogram was performed to assess the patency of the artificial atrial septal defect. Selected tracings from the film sequence are shown in Figures 3 and 4 . Figure 3 shows the patient in the left anterior oblique position. Figure $3 \mathrm{~A}$ shows the right atrium. The large atrial septal defect with filling of the left atrium can be seen in Figure 3B. Figure 3C shows the large anteriorly situated aorta arising from the right ventricle, and Figure 3D demonstrates good filling of the left atrium and ventricle and pulmonary artery. There was good mixing of the systemic and pulmonary venous return and no pulmonary stenosis. In addition intermitten opacification of the right atrium suggested that there was a left to right shunt during part of the cardiac cycle with a right to left shunt during the remainde옹 of the cycle. Figure 4 shows the same sequence in the right anterior oblique position, confirming good. mixing between the right and left atria.

\section{DISCUSSION}

In this patient with transposition of the greak vessels and an intact ventricular septum, clinicat deterioration coincided with closure of the ductu露 arteriosus. Adequate mixing of the systemic and pulmonary circuits became impossible. Although the creation of an atrial septal defect did not curE్ the underlying condition, the effect of palliative surgery was dramatic and the post-operative cinéangiocardiogram showed a large bidirectiona $\Phi$ 

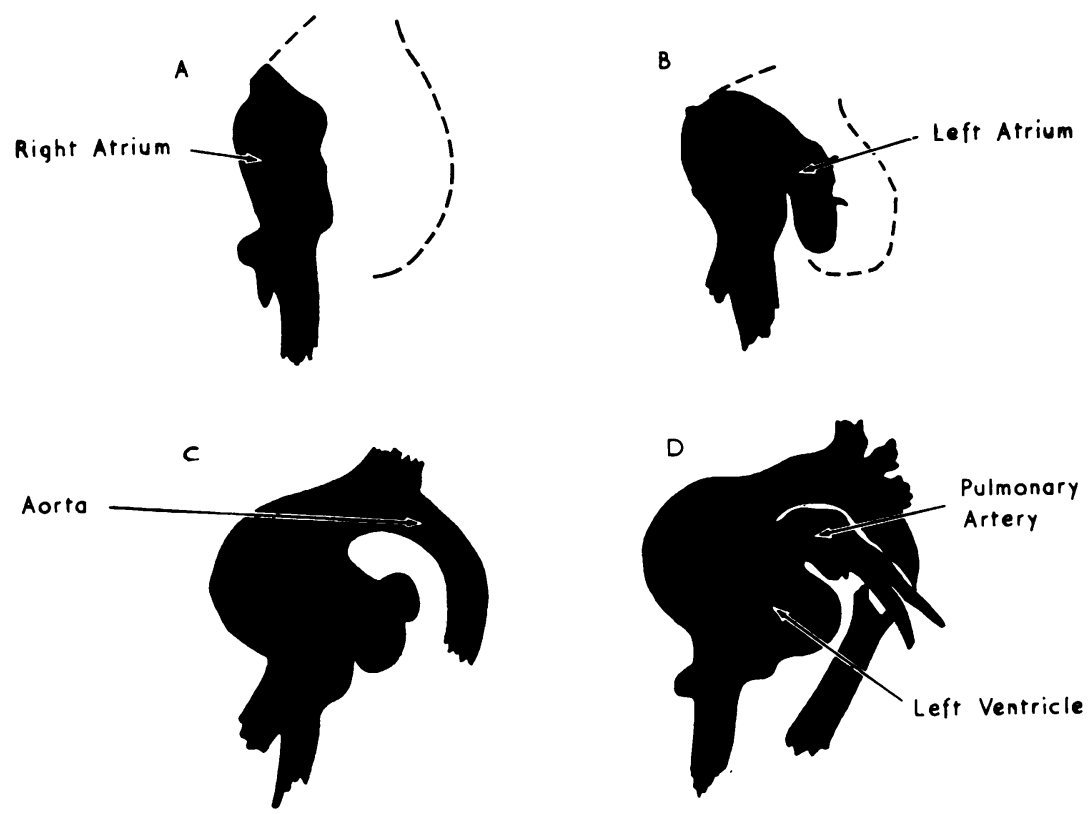

FIG. 3. Venous cinéangiocardiogram after operation-left anterior oblique position. (A) Contrast medium entered the right atrium and refluxed into the hepatic veins. (B) Contrast medium passed across the large atrial septal defect and filled the left atrium. A dilution defect was seen in the right atrium during part of the cardiac cycle, confirming the presence of a bidirectional shunt across the defect. $(C)$ Contrast entered the anteriorly situated aorta which arose from the right ventricle. The left atrium and ventricle were well filled. (D) Opacification of the pulmonary artery from the left ventricle was nearly as dense as opacification of the aorta. There was therefore a large right to left shunt through the newly created atrial septal defect.
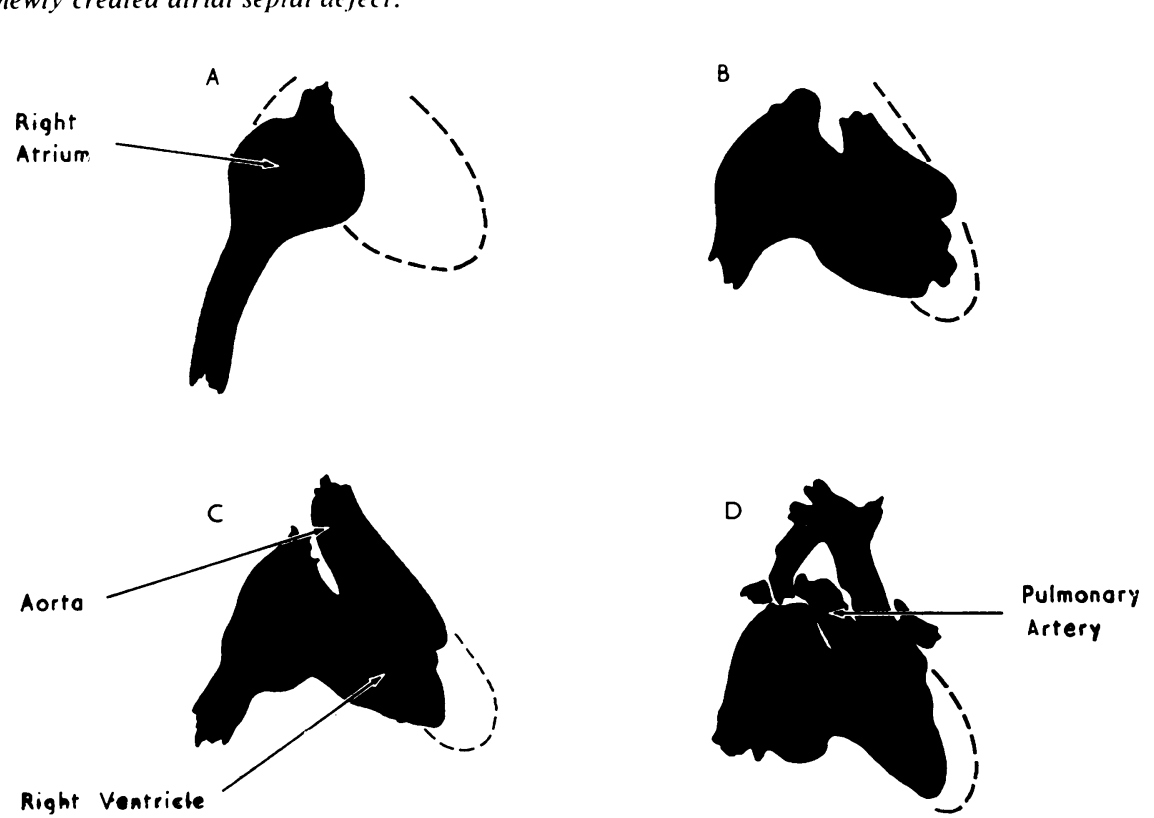

FIG. 4. Venous cinéangiocardiogram after operation-right anterior oblique position. (A) The right atrium filled. (B) The right ventricle filled. Simultaneous opacification of the left atrium and ventricle were not visible in this view. (C) Early opacification of the aorta from the right ventricle. (D) Simultaneous filling of the aorta and pulmonary artery, indicating a good pulmonary blood flow from the systemic venous return.

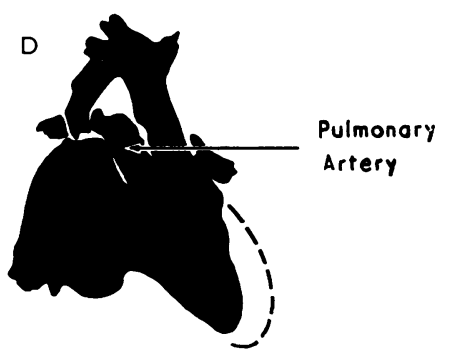


shunt with adequate mixing through a nonrestrictive atrial septal defect. The patient is still alive and well six months after operation.

Ochsner et al. (1961) have reported an increase in oxygen saturation and a similar improvement in clinical status after this procedure, and, although Trusler et al. (1964) report a high mortality from operation in this group of critically ill infants, the survivors improved. Our patient showed a gratifying and satisfying response to surgery. The operation improves the physiological status of the circulation, and with earlier diagnosis, a more aggressive policy of treatment, and better post-operative care, further improvement can be expected in this difficult group of neonates.

I am grateful to Drs. Clifford Parsons and Roy Astley for encouragement, to Mr. L. D. Abrams for operating on the child, and to Mrs. C. M. Hall for secretarial assistance.

\section{REFERENCES}

Abbott, M. E. (1936). Atlas of Congenital Cardiac Disease. American Heart Association, New York.

Blalock, A., and Hanlon, C. R. (1950). The surgical treatment complete transposition of the aorta and the pulmonary arter,
Surg. Gynec. Obstet., 90, 1 .

Fontana, R. S., and Edwards, J. E. (1962). Congenital Cardiant Disease. W. B. Saunders, Philadelphia.

Hanlon, C. R., and Blalock, A. (1948). Complete transposition of the aorta and the pulmonary artery. Ann. Surg., 127, 385.

Keith, J. D., Neill, C. A., Vlad, P., Rowe, R. D., and Chute, A. (1953). Transposition of the great vessels. Circulation, 7, 830. Rowe, R. D., and Vlad, P. (1958). Heart Disease in Infancy an Childhood. Macmillan, New York.

Mustard, W. T. (1964). Successful two-stage correction of $\operatorname{tran}$ position of the great vessels. Surgery, 55, 469.

Keith, J. D., Trusler, G. A., Fowler, R , and Kidd, L. (1964 The surgical management of transposition of the great vessels $J$. thorac. cardiovasc. Surg., 48, 953.

Noonan, J. A., Nadas, A. S., Rudolph, A. M., and Harris, G. C. B? (1960). Transposition of the great arteries. A correlation of clinical, physiologic and autopsy data. New Engl. J. Med. 263 $592,637,684,739$.

Ochsner, J. L., Cooley, D. A., Harris, L. C., and McNamara, D. GO (1961). Treatment of complete transposition of the great vessel with the Blalock-Hanlon operation. Circulation, 24, 51.

Trusler, G. A., Mustard, W. T., and Fowler, R. S. (1964). The role oठ̂ surgery in the treatment of transposition of the great vessels $\mathbb{Q}$ Canad. med. Ass. J., 91, 1096. 